\title{
Star-shaped Local Density of States around Vortices in a Type II Superconductor
}

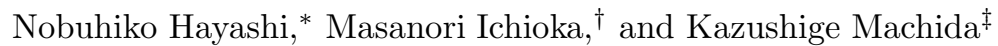 \\ Department of Physics, Okayama University, \\ Okayama 700, Japan
}

\begin{abstract}
The electronic structure of vortices in a type II superconductor is analyzed within the quasi-classical Eilenberger framework. The possible origin of a sixfold "star" shape of the local density of states, observed by scanning tunneling microscope experiments on $\mathrm{NbSe}_{2}$, is examined in the light of the three effects; the anisotropic pairing, the vortex lattice, and the anisotropic density of states at the Fermi surface. Outstanding features of split parallel rays of this star are well explained in terms of an anisotropic $s$-wave pairing. This reveals a rich internal electronic structure associated with a vortex core.
\end{abstract}

Much attention has been focused on a vortex structure in high $T_{c}$ cuprate superconductors, yet some of fundamental problems associated with vortices still remain even in conventional superconductors. The electronic structure around a vortex core is one of them. Notably, Hess et al. [1 6 ] have done a series of remarkable and beautiful scanning tunneling microscope (STM) experiments on a layered hexagonal compound $2 \mathrm{H}-\mathrm{NbSe}_{2}$ $\left(T_{c}=7.3 \mathrm{~K}\right)$ to reveal the detailed spatially resolved electronic structure around a vortex core. They provide direct and vivid images of individual vortices and the flux line lattice. It was shown theoretically that the STM images observed by Hess et al. and Renner et al. [7] reflect the local density of states (LDOS) for bound states around a vortex. On the basis of the Bogoliubov-de Gennes (BdG) theory 810] or the quasi-classical Eilenberger (QCE) theory [11,12], the case of the cylindrical symmetric isolated vortex was considered there. However, the detailed structure of the LDOS observed is not fully understood.

The outstanding features of the experimental findings [2 6] are summarized as follows when the magnetic field $H$ is applied perpendicular to the hexagonal plane: (1) The LDOS for quasiparticle excitations has a sixfold "star" shape centered at a core. (2) The orientation of this star depends on the bias energy. At zero bias the "ray" extends away from the nearest-neighbor vortex direction [Fig. 1(a)] where the conventional $60^{\circ}$ Abrikosov vortex lattice is formed. Upon increasing the bias voltage the star rotates by $30^{\circ}$ [Fig. 11(c)]. (3) In the intermediate bias voltage a ray splits into a pair of nearly parallel rays, keeping its direction fixed [Fig. 11(b)]. (4) In the spectral evolution as shown in Fig. 9 in Ref. [6] (also see Fig. 6 in Ref. (3)) there exist additional inner ridges other than the outer ridges evolving from the zero bias peak at a core into the bulk BCS like gap edges far from a core.

To understand these experimental results, Gygi and Schlüter 10,13 discussed the sixfold symmetric structure on the basis of the BdG theory by introducing a sixfold symmetric perturbation term and using their numerical solutions of the cylindrical symmetric case. While they explained the above mentioned features (1) and (2), the origin of their perturbation term is not clear, and it is uncertain whether the sixfold symmetric term can be treated by a perturbation theory. We notice that the features (3) and (4) are difficult to be explained in their framework. In the following, we show that an anisotropic pairing (or gap) effect which has been ignored so far is crucial for the bound states around a vortex.

The purpose of this paper is to clarify the origin of the characteristic star-shaped LDOS by the QCE theory [14. The QCE approach is more suitable than the BdG approach for the case cylindrical symmetry is broken. The anisotropic pairing can be treated easily by the QCE theory. As for the origin of the sixfold symmetric structure, the following possibilities are enumerated; (A) the effect of an anisotropic pairing, (B) the effect of nearest-neighbor vortices, that is, the effect of the vortex lattice, and (C) the effect of the anisotropic density of states at the Fermi surface (FS). We calculate the LDOS for each case (A)-(C), and investigate which case can explain the LDOS of the STM experiments.

We introduce an anisotropic $s$-wave pairing $\Delta(\mathbf{r}, \mathbf{k})=$ $\Delta_{0}(\mathbf{r}) F(\theta)=\Delta_{0}(\mathbf{r})\left(1+c_{\mathrm{A}} \cos 6 \theta\right)$ with hexagonal symmetry and assume two-dimensional circular FS, neglecting a small warping along the hexagonal $z$ axis $(\| \mathbf{H})$, which is appropriate to $\mathrm{NbSe}_{2}$. Here $\mathbf{r}=(x, y)$ is the center of mass coordinate and $\mathbf{k}$ the relative coordinate of a Cooper pair. Now $\mathbf{k}$ is denoted by an angle $\theta$ measured from the $a$ axis (or $x$ axis) in the hexagonal plane. Thus the parameter $c_{\mathrm{A}}$ denotes the degree of anisotropy in a pairing [15, 16. The case $c_{\mathrm{A}}=0$ corresponds to a conventional isotropic pairing.

Our calculation is performed after the method of Ref. 117] for the isolated vortex and that of Klein 18, 19 for the vortex lattice. We consider a transport-like Eilenberger equation [14] for the quasi-classical Green function $\hat{g}\left(\mathbf{r}, \theta, i \omega_{n}\right)=-i \pi\left(\begin{array}{cc}g & i f \\ -i f^{\dagger} & -g\end{array}\right)$ in a $2 \times 2$ matrix form, namely,

$$
\begin{aligned}
i \mathbf{v}_{\mathrm{F}} & \cdot \nabla \hat{g}\left(\mathbf{r}, \theta, i \omega_{n}\right) \\
& +\left[\left(\begin{array}{cc}
i \omega_{n} & -\Delta(\mathbf{r}, \theta) \\
\Delta^{*}(\mathbf{r}, \theta) & -i \omega_{n}
\end{array}\right), \quad \hat{g}\left(\mathbf{r}, \theta, i \omega_{n}\right)\right]=0
\end{aligned}
$$


supplemented by the normalization condition: $\hat{g}\left(\mathbf{r}, \theta, i \omega_{n}\right)^{2}=-\pi^{2} \hat{1}$. The Fermi velocity is $v_{\mathrm{F}}$. The bracket [ , ] is a commutator. The self-consistent equation is given by

$$
\Delta(\mathbf{r}, \theta)=2 \pi T \sum_{\omega_{n}>0} \int \frac{d \theta^{\prime}}{2 \pi} \rho\left(\theta^{\prime}\right) V\left(\theta, \theta^{\prime}\right) f\left(\mathbf{r}, \theta^{\prime}, i \omega_{n}\right),
$$

where the pairing interaction $V\left(\theta, \theta^{\prime}\right)=v F(\theta) F\left(\theta^{\prime}\right)$ is assumed to be separable. The $\theta$-dependent density of states at the FS is $\rho(\theta)$. We calculate $\Delta_{0}(\mathbf{r})$ self-consistently from Eqs. (1) and (2) for $T / T_{c}=0.1$. The LDOS is given by

$$
\begin{aligned}
N(\mathbf{r}, E) & =\int_{0}^{2 \pi} \frac{d \theta}{2 \pi} \rho(\theta) N(\mathbf{r}, \theta, E) \\
& =\int_{0}^{2 \pi} \frac{d \theta}{2 \pi} \rho(\theta) \operatorname{Re} g\left(\mathbf{r}, \theta, i \omega_{n} \rightarrow E+i \eta\right),
\end{aligned}
$$

where $\eta(>0)$ is an infinitesimal constant. To obtain $g\left(\mathbf{r}, \theta, i \omega_{n} \rightarrow E+i \eta\right)$, we solve Eq. (1) for $\eta-i E$ instead of the Matsubara frequency $\omega_{n}$ using the self-consistently obtained pair potential. From now on, the energy $E$ and the length $r$ are scaled by the uniform gap $\Delta_{0}$ at zero temperature and $\xi=v_{\mathrm{F}} / \Delta_{0}$ respectively.

Let us start out by considering (A) the effect of an anisotropic pairing. The anisotropic pairing is suggested from the STM experiment at zero field. The observed $I-V$ tunneling spectrum in the bulk indicates a substantial gap anisotropy (the gap amplitude with the averaged value $1.1 \mathrm{mV}$ distributes from $0.7 \mathrm{mV}$ to $1.4 \mathrm{mV}$, see Fig.1 in Ref. [3]), which is consistent with the density of states in the anisotropic pairing case (see Fig. 5 in Ref. [16]). To see the influence of (A) clearly, we neglect the other effects (B) and (C) here. We calculate the LDOS in the isolated vortex case assuming an isotropic FS.

In Figs. 1(d), 1(e), and 1(f), we show the calculated LDOS for several bias energies $E$ in the case $c_{\mathrm{A}}=0.5$. It is seen from Fig. 1 1 (d) that the sixfold star centered at a core is oriented away from the $x$ axis by $30^{\circ}$ for $E=0$. The rays along the $30^{\circ}$ direction can be seen to extend up to $r \sim 3$ from the center. It corresponds to the following fact. As for the bound state around a vortex core at $E=0$, the wave function of the quasiparticle running through the vortex center has the maximum amplitude. Among them, because $F(\theta)$ is most depressed at $\theta=30^{\circ}$, the quasiparticle with the momentum $\mathbf{k}$ along the $30^{\circ}$ direction feels weakest superconducting pair potential, and its wave function keeps large amplitude until far from the vortex.

It is seen from Fig. 11(e) that at the intermediate energy each ray splits into two parallel rays, keeping its direction. This characteristic feature is precisely observed by Hess [Fig. 11(b)] (also see Refs. [4 5]) and is absent in the result by Gygi and Schlüter [10,13]. For this intermediate excitation energy, the wave function of the quasiparticle passing at a small finite distance from the vortex center has the maximum amplitude. Among them, the quasiparticle with the momentum $\mathbf{k}$ along the $30^{\circ}$ and its equivalent directions keeps large amplitude until far from the vortex, and forms the nearly parallel structure in the $\mathbf{r}$ space.

With increasing the bias energy $E$ further, the sixfold star becomes a more extended one, and its orientation rotates by $30^{\circ}$ as seen from Fig. 1(f). Note that the heads of each ray spread out. It coincides with the observation in Fig. 11(c).

Another way to examine the quasiparticle excitations in the vortex state is to see how the spectrum evolves along the radial lines. The calculated spectrum evolution shown in Fig. 2 is compared with the data in Fig. 9 in Ref. [6] (also see Fig. 6 in Ref. [3]). Here the radial line is chosen to make an angle $15^{\circ}$ from the $x$ axis, where there are five ridges for $E>0$. We label these ridges as $\alpha-\varepsilon$. As $r$ increases, two higher energy ridges $\alpha$ and $\beta$ approach the maximum of the gap energy $1+c_{\mathrm{A}}$, and the ridge $\gamma$ is found to become very weak intensity. The ridge $\delta$, which approaches the minimum of the gap energy $1-c_{\mathrm{A}}$ with increasing $r$, corresponds to that reported in the isotropic pairing model by Gygi and Schlüter (see Fig. 15 in Ref. 10 ). The ridge $\varepsilon$ is the result reflecting the sixfold symmetric ray structure. The ridges $\delta$ and $\varepsilon$ correspond to the outer and inner ridges in the experimental data [3,6] by Hess et al., respectively.

To clarify the behavior of these ridges, we show in Fig. 3 the trace of the ridges in the LDOS as a function of $r$ along the radial lines for $0^{\circ}(\mathrm{a}), 15^{\circ}(\mathrm{b})$, and $30^{\circ}(\mathrm{c})$ from the $x$ axis. For comparison, the experimental data [3] are also presented. We direct our attention to the inner ridge (line $\varepsilon$ ), which strongly depends on the radial angle. It moves toward the lower energy side as the angle increases. At $30^{\circ}$, it reduces to the ridge at $E=0$ (horizontal $r$ axis). This behavior of the inner ridge agrees well with that of the experimental data.

Thus, the effect of the anisotropic pairing can explain not only (1) the sixfold star shape and (2) the $30^{\circ}$ rotation, but also (3) the split parallel ray structure at the intermediate energy and (4) the characteristic behavior of the inner ridges in the spectral evolution.

Notice that this characteristic spreading of the ray structure depends on the degree of anisotropy $c_{\mathrm{A}}$; In the isotropic limit $\left(c_{\mathrm{A}}=0\right)$ the star shape turns into a circular object. As $c_{\mathrm{A}}$ increases, the star structure spreads out farther from a core. This implies that in $\mathrm{NbSe}_{2}$ a substantial gap anisotropy must exist even within the hexagonal plane. It is consistent with the tunneling experiment 3] in the bulk mentioned earlier.

Let us argue a possible origin of this rather strong gap anisotropy. It is known that the system enters into a charge density wave (CDW) state at $32 \mathrm{~K}$ which coexists with superconductivity at lower temperatures. The FS's consist of two kinds of cylinders, each centered at the $\Gamma$ point and $K$ point in reciprocal space 20]. The CDW re- 
organizes the FS's, opening up the CDW gaps by nesting. The remaining FS's left over by the CDW gap formation are available for the superconductivity. Therefore, we can expect a substantial gap anisotropy within the hexagonal plane.

Next, we turn to (B) the effect of the vortex lattice. The LDOS is calculated in the case of a triangular vortex lattice by using the self-consistently obtained pair potential. Here, neglecting the other effects $(\mathrm{A})$ and $(\mathrm{C})$, we consider the isotropic $s$-wave pairing $\left(c_{\mathrm{A}}=0\right)$ and assume an isotropic FS. As for the LDOS in the vortex lattice case, Klein calculated only the momentum-resolved LDOS $N(\mathbf{r}, \theta, E)$ in Eq. (3) for specific $\mathbf{k}$ direction parallel to $m \mathbf{r}_{1}+n \mathbf{r}_{2}$ ( $m$ and $n$ are integer, $\mathbf{r}_{1}$ and $\mathbf{r}_{2}$ are unit vectors of the vortex lattice) because the so-called symmetric method was used 19. Using the explosion method 17 19, we succeed to calculate $N(\mathbf{r}, \theta, E)$ for arbitrary $\mathbf{k}$ direction. Then we can obtain the LDOS integrated over $\mathbf{k}$ directions.

Important results obtained for the vortex lattice are; (i) We do find a sixfold star shape of the LDOS and the $30^{\circ}$ rotation upon elevating the bias energy. The sixfold star originates from the triangular vortex lattice effect. (ii) The orientation of the star coincides with the STM data, namely the ray extends toward the next nearestneighbor vortex direction at lower bias energy. Therefore we succeed in determining the absolute direction relative to the flux lattice. This is one of the most eminent features in the STM data and absent in Gygi and Schlüter [10,13. (iii) However, the split parallel ray structure at the intermediate energy is not reproduced. (iv) The characteristic sixfold symmetric LDOS appears only at a high magnetic field such as 1 Tesla for the material parameters appropriate to $\mathrm{NbSe}_{2}$ (The BCS coherence length $77 \AA$ and the BCS penetration depth $690 \AA$ [3]), where the core regions substantially overlap each other. At a lower magnetic field such as 0.1 Tesla, the LDOS reduces to the almost circular structure.

As for (C) the effect of the anisotropic density of states at the FS, we find that reflecting the assumed sixfold anisotropy the LDOS exhibits the characteristic star shape and the $30^{\circ}$ rotation even for isotropic s-pairing. However, the split parallel ray structure at the intermediate energy is not reproduced.

In conclusion, by solving the quasi-classical Eilenberger equation self-consistently, we have examined the possible origins of the star-shaped LDOS observed in the STM experiments on $\mathrm{NbSe}_{2}$. Among the three effects (A), (B), and $(\mathrm{C})$, each one can reproduce the experimental features (1) the sixfold star shape and (2) the $30^{\circ}$ rotation. In $\mathrm{NbSe}_{2}$, all the three effects may play important roles. However, to reproduce the features (3) the split parallel ray structure at the intermediate energy and (4) the characteristic behavior of the inner ridges in the spectral evolution, we have to consider the LDOS by including the effect of the anisotropic pairing.
As for the relation between the orientation of the star shape and that of the vortex lattice, the calculation for the vortex lattice has to be done by including the anisotropy effect. It is noted that the orientations of the star shape and of the vortex lattice relative to the underlying crystal lattice are internally correlated through the gap function. The gap function itself is determined by the FS topology. The complete answer to this can be obtained by considering all the three effects simultaneously, which belongs to a future work. The present study underlies the basic understanding for fundamental electronic properties of vortices both for conventional and unconventional superconductors, revealing a rich internal electronic structure associated with vortices.

In connection to the anisotropic pairing effect, we point out that high $T_{c}$ superconductors should exhibit similar STM characteristics if a $d$-wave pairing such as $\Delta(\mathbf{r}, \mathbf{k})=$ $\Delta_{0}(\mathbf{r}) \cos 2 \theta$ is realized. We predict a "fourfold" star and its $45^{\circ}$ rotation there [17].

We would like to thank H. F. Hess for providing unpublished data and the original photographs for reproducion, and for encouragement, which certainly motivates the present project.

* Electronic address: hayashi@mp.okayama-u.ac.jp

† Electronic address: oka@mp.okayama-u.ac.jp

¥ Electronic address: machida@mp.okayama-u.ac.jp

[1] H. F. Hess et al., Phys. Rev. Lett. 62, 214 (1989).

[2] H. F. Hess, R. B. Robinson, and J. V. Waszczak, Phys. Rev. Lett. 64, 2711 (1990).

[3] H. F. Hess, R. B. Robinson, and J. V. Waszczak, Physica B 169, 422 (1991).

[4] H. F. Hess, Physica C 185-189, 259 (1991).

[5] H. F. Hess, Jpn. J. Appl. Phys. Series 9, 270 (1993).

[6] H. F. Hess, in Scanning Tunnering Microscopy, ed. J. A. Stroscio and W. J. Kaiser (Academic Press, San Diego, 1993) p.427.

[7] Ch. Renner et al., Phys. Rev. Lett. 67, 1650 (1991).

[8] J. D. Shore, M. Huang, A. T. Dorsey, and J. P. Sethna, Phys. Rev. Lett. 62, 3089 (1989).

[9] F. Gygi and M. Schlüter, Phys. Rev. B 41, 822 (1990).

[10] F. Gygi and M. Schlüter, Phys. Rev. B 43, 7609 (1991).

[11] U. Klein, Phys. Rev. B 41, 4819 (1990).

[12] S. Ullah, A. T. Dorsey, and L. J. Buchholtz, Phys. Rev. B 42, 9950 (1990).

[13] F. Gygi and M. Schlüter, Phys. Rev. Lett. 65, 1820 (1990).

[14] G. Eilenberger, Z. Phys. 214, 195 (1968).

[15] D. Markowitz and L. P. Kadanoff, Phys. Rev. 131, 563 (1963); J. R. Clem, Phys. Rev. 148, 392 (1966).

[16] J. R. Clem, Ann. Phys. (N.Y.) 40, 268 (1966).

[17] M. Ichioka, N. Hayashi, N. Enomoto, and K. Machida, Phys. Rev. B 53, 15316 (1996). 
[18] U. Klein, J. Low Temp. Phys. 69, 1 (1987).

[19] U. Klein, Phys. Rev. B 40, 6601 (1989).

[20] See for example, R. Corcoran et al., J. Phys. C6, 4479 (1994).

FIG. 1. Tunneling conductance images observed by Hess et al. at 0.1 Tesla for the bias voltage $0.0 \mathrm{mV}(\mathrm{a}), 0.24 \mathrm{mV}(\mathrm{b})$, $0.48 \mathrm{mV}$ (c), where $1759 \AA \times 1759 \AA$ is shown (also see Refs. $[4,5])$. The nearest-neighbor direction of the vortex lattice is the horizontal direction. The LDOS images calculated for $E=0(\mathrm{~d}), 0.2(\mathrm{e})$, and $0.32(\mathrm{f})$, where $6 \xi \times 6 \xi$ is shown.

FIG. 2. Evolution of the spectra along a radial line at the angle $15^{\circ}$ from the $x$ axis. Each ridge is labeled as $\alpha-\varepsilon$ from the high energy side.

FIG. 3. The ridge energies of the LDOS as a function of $r$ along the radial lines for $0^{\circ}$ (a), $15^{\circ}$ (b), and $30^{\circ}$ (c) from the $x$ axis (solid lines). The case (b) corresponds to Fig. 2, where the labels $\alpha-\varepsilon$ are the same as those in Fig. 2. Experimental data[3] are also presented by points $\bullet$ (outer) and $\circ$ (inner), where $r$ and $E$ are scaled by $350 \AA$ and $1.67 \mathrm{mV}$, respectively. 